\title{
(Precursor for) Quantum Boundary Conditions for Expanding Universe
}

\author{
Andrew Walcott Beckwith \\ Physics Department, College of Physics, Chongqing University Huxi Campus, Chongqing, China \\ Email: rwill9955b@gmail.com, abeckwith@uh.edu
}

How to cite this paper: Beckwith, A.W. (2017) (Precursor for) Quantum Boundary Conditions for Expanding Universe. Journal of High Energy Physics, Gravitation and Cosmology, 3, 16-20.

http://dx.doi.org/10.4236/jhepgc.2017.31003

Received: October 6, 2016

Accepted: November 19, 2016

Published: November 23, 2016

Copyright $\odot 2017$ by author and Scientific Research Publishing Inc. This work is licensed under the Creative Commons Attribution International License (CC BY 4.0).

http://creativecommons.org/licenses/by/4.0/ cc) (i) Open Access

\begin{abstract}
Using Hall and Reginatto's condition for a Wheeler De Witt Equation for a Friedman-Walker metric coupled to a (Inflaton) scalar field $\phi$, we delineate the outer boundary of the value of a scale factor $a(t)$ for quantum effects, in an expanding universe. The inflaton field is from Padmanabhan's reference, “An Invitation to Astrophysics" which yields a nonstandard Potential $U(a, \phi)$ which will lead to an algebraic expression for $a(t)$ for the value of the outer boundary of quantum effects in the universe. Afterwards, using the scale factor $a(t)=a_{\text {initial }} \cdot t^{\alpha}$, with alpha given different values, we give an estimation as to a time, $t$ (time) which is roughly the boundary of the range of quantum effects. How this is unusual? We use the Wheeler De Witt Equation, as a coupling to a given inflaton field $\phi$ and find a different way as to delineate a time regime for the range of quantum effects in an expanding universe.
\end{abstract}

\section{Keywords}

Wheeler De Witt Equation, Inflaton, Friedman-Walker Metric, Scale Fact

\section{Introduction}

We work with the Wheeler De Witt Equation as given by [1], as part of the work by Hall and Reginatto, in 2016, where an ordering, called $p$, is used to link a Wheeler De Witt Equation, as given below, to an inflaton, and the Friedman Walker space-time metric, with the inflaton described by [2] and the Friedman Walker metric given in [2] [3].

What we are doing is using [1] with its Wheeler De Witt equation to look at the following

$$
\left[\frac{\partial^{2}}{\partial a^{2}}+\frac{p}{a} \frac{\partial}{\partial a}-\frac{1}{a^{2}} \frac{\partial^{2}}{\partial \phi^{2}}-U(a, \phi)\right] \Psi=0
$$


The inflaton, $\phi$ is defined by [2] as given by

$$
\begin{aligned}
& \phi=\sqrt{\frac{\alpha}{4 \pi G}} \ln \left(\sqrt{\frac{8 \pi G V_{0}}{\alpha \cdot(3 \alpha-1)}} t\right) \\
& U(a, \phi)=V_{0} \exp \left(-\sqrt{\frac{16 \pi G}{\alpha}} \phi\right) \\
& a=a_{\text {initial }} t^{\alpha}
\end{aligned}
$$

The wave function we use in Equation (1) we will use the ansatz of

$$
\Psi=\Psi_{\text {initial }} \exp (\beta \cdot a(t) \phi(t))
$$

These three sets of equations will be referenced, in our article, and will form the template of the subsequent analysis.

\section{Looking at How to Come Up with a Polynomial} Equation for $a(t)$

$a(t)$ as given in Equation (2) is used to re define the inflaton in Equation (2) as well as a re definition of the potential $U$, as in Equation (2) with the upshot that

$$
\begin{aligned}
& \left.\phi\right|_{\text {redone }}=\sqrt{\frac{\alpha}{4 \pi G}} \ln \left(\sqrt{\frac{8 \pi G V_{0}}{\alpha \cdot(3 \alpha-1)}} \cdot\left(\frac{a_{\text {initial }}}{a}\right)^{1 / \alpha}\right) \\
& \left.U(a, \phi)\right|_{\text {redone }}=V_{0}\left(\frac{8 \pi G}{\alpha \cdot(3 \alpha-1)}\right)^{-1} \cdot\left(\frac{a_{\text {initial }}}{a}\right)^{2 / \alpha} \\
& p=-1, \alpha=1 \\
& \Rightarrow a^{2}-a \cdot\left(\frac{\phi(t)}{\beta \cdot\left(1-\beta^{2}\right)}\right)-\frac{V_{o} \cdot a_{\text {initial }}}{4 \pi G}=0
\end{aligned}
$$

Now what is unusual about the bottom quadratic equation for the scale factor, as given in Equation (4)? We have that, here we are using Equation (2) in the end to define, here, a $\phi$ inflaton equation in terms of time, not the scale factor version of it, as given in Equation (4). If we use this approach, and constrain ourselves to very small time steps, i.e. of the order of Planck scale time (very small) we get then that the range of quantum effects, from an initial $a_{\text {initial }}$ to the boundary of quantum gravity effects, is given by, approximately for small $a_{\text {initial }}$.

$$
a(\text { quantum-outer-boundary }) \sim\left(\frac{1}{2} \cdot \frac{\beta \cdot\left(1-\beta^{2}\right) V_{0}}{\pi G \cdot\left|\phi_{\text {initial }}\right|}\right) \cdot a_{\text {initial }}
$$

\section{Conclusion: We Have Taken the Simplest Case, and It Could Be More Complicated}

What we have done is to look at $\alpha=1$, while using the inflaton expression given in Equation (6) below:

$$
\phi(\text { redone })=\sqrt{\frac{\alpha}{4 \pi G}} \ln \left(\sqrt{\frac{8 \pi G V_{0}}{\alpha \cdot(3 \alpha-1)}} \cdot\left(\frac{a_{\text {initial }}}{a}\right)^{1 / \alpha}\right)
$$


In

$$
a^{2}-a \cdot\left(\frac{\phi(t)}{\beta \cdot\left(1-\beta^{2}\right)}\right)-\frac{V_{o} \cdot a_{\text {initial }}}{4 \pi G}=0
$$

Were we to insert Equation (6) for the inflaton into Equation (7) we would have a very nonlinear case, for the scale factor equation. One which could only be deciphered by numerical analysis.

If we stick with the above methodology, we still have to consider conditions for which

$$
a(\text { quantum-outer-boundary }) \sim\left(\frac{1}{2} \cdot \frac{\beta \cdot\left(1-\beta^{2}\right) V_{0}}{\pi G \cdot\left|\phi_{\text {initial }}\right|}\right) \cdot a_{\text {initial }}
$$

Which presumably would be linked to

$$
\left(\frac{1}{2} \cdot \frac{\beta \cdot\left(1-\beta^{2}\right) V_{0}}{\pi G \cdot\left|\phi_{\text {initial }}\right|}\right)>1
$$

Indeed, though, if $\alpha \neq 1$ there is no way we could possibly retrieve Equation (4) above, i.e. we have a numerical problem, one which we will investigate in future papers. In addition, for Equation (4), Equation (7) and Equation (8) we need to remember $\beta$ comes from Equation (3) and its value will need to be considered.

What we have though is based upon [1] and the idea of a quantum ensemble and operator-ordering. In order for the readers to get more insights as to the physics inherent in the choice of $p$, in Equation (1) the reader is referred to [4] [5] [6].

Finally, [7]-[12] have issues which need to be reviewed which may in fact, have a ready impact upon Equation (8), and Equation (9) above, i.e. [7] [8] [9] refers to Corda's work with the foundation of gravity, and if or not Gravity is quantum, or purely due to classical General Relativity. In particular the issue of scalar-tensor gravity needs to be investigated, to see if it falsifies Equation (7) or if it adds new restrictions as to the boundaries.

Note also, that [10] touches upon if or not quantum mechanics is part of a deterministic set up, which would have immediate consequences as to Equation (3). References [11] [12] as to higher dimensions, should be looked at as far as the fidelity of Equation (1) to the setup of the universe concluded. I.e. both references postulate higher dimensions. In addition $\mathrm{Ng}$ [13] have it that there would be a wavelength, as part of the derivation of entropy included in the entropy formula of

$$
S \sim N(\text { particle-count }) \times\left(\ln \left(V / \lambda^{3}\right)+3 / 2\right)
$$

The answer, as given by $\mathrm{Ng}$, is that if the volume of space, $V$, is- $\lambda^{3}$, and that $\lambda$ is proportional to the wavelength, then due to the situation of how a massive graviton could at least have accelerated mass values, this will allow for the $\mathrm{Ng}$ formula, being changed to

$$
S \sim N(\text { particle-count })
$$

Does Equation (8) and Equation (9) falsify Equation (10) and Equation (11)? 
It needs to be answered. And of course all this needs to avoid being in conflict with [14] and the gravity results so derived. Finally, does Equation (8) and Equation (9), not to mention Equation (4) falsify the conditions given in [15] as to massive gravity? This question should also be investigated.

After these questions are entertained, and examined, the last supposition, as mentioned should be investigated, i.e. of a different time variable, delineating the amount of time in a quantum regime for the expansion of the universe. IMO, using

$$
a=a_{\text {initial }} t^{\alpha}
$$

And if $\alpha \sim 1$ would lead to a time regime for quantum effects, delineated by

$$
t \text { (quantum-outer-boundary) } \sim\left(\frac{1}{2} \cdot \frac{\beta \cdot\left(1-\beta^{2}\right) V_{0}}{\pi G \cdot\left|\phi_{\text {initial }}\right|}\right)
$$

Of course, if $\alpha \neq 1$, we would have a different power relationship, very different.

I.e. all these questions need to be investigated in the near future.

\section{Acknowledgements}

This work is supported in part by National Nature Science Foundation of China grant No. 11375279.

\section{References}

[1] Hall, M.J.W. and Reginatto, M. (2016) Ensembles on Configuration Space, Classical, Quantum, and Beyond. Fundamental Theories of Physics, Volume 184, Springer Verlag, Springer International Series Publishing, Geneva.

[2] Padmanabhan, T. (2006) An Invitation to Astrophysics. World Scientific Series in Astronomy and Astrophysics, Volume 8, World Scientific Publishing Company, Pte. Ltd., 5 Toh Tuck Link, Singapore.

[3] Kieffer, K. (2012) Quantum Gravity. 3rd Edition, Oxford University Press, Oxford, UK.

[4] Kontoleon, N. and Wiltshire, D. (1999) Operator Ordering and Consistency of the Wave Function of the Universe. Physical Review D, 59, Article ID: 063513. https:/doi.org/10.1103/PhysRevD.59.063513

[5] Vilenkin, A. (1986) Boundary Conditions in Quantum Cosmology. Physical Review D, 33, 3560-3569. https:/doi.org/10.1103/PhysRevD.33.3560

[6] Hawking, S. and Page, D. (1986) Operator Ordering and the Flatness of the Universe. Nuclear Physics B, 264, 185-196. https:/doi.org/10.1016/0550-3213(86)90478-5

[7] Corda, C. (2008) Primordial Production of Massive Relic Gravitational Waves from a Weak Modification of General Relativity. Astroparticle Physics, 30, 209-215.

http://arxiv.org/abs/0812.0483 https:/doi.org/10.1016/j.astropartphys.2008.09.003

[8] Corda, C. (2009) Interferometric Detection of Gravitational Waves: The Definitive Test for General Relativity. International Journal of Modern Physics D, 18, 2275-2282. http://arxiv.org/abs/0905.2502 https:/doi.org/10.1142/s0218271809015904

[9] Corda, C. (2007) A Longitudinal Component in Massive Gravitational Waves Arising from a bimetric Theory of Gravity. Astroparticle Physics, 28, 247-250.

http://arxiv.org/abs/0811.0985

https:/doi.org/10.1016/j.astropartphys.2007.05.009 
[10] t' Hooft, G. (2007) Emergent Quantum Mechanics and Emergent Symmetries. AIP Conf. Proc., 957, 154-163. https://arxiv.org/abs/0707.4568

[11] Arkani-Hamed, N., Dimopoulos, S., Kaloper,N. and Sundrum, R. (2000) A Small Cosmological Constant from a Large Extra Dimension. Physics Letters B, 480, 193-199.

https://arxiv.org/abs/hep-th/0001197 https:/doi.org/10.1016/S0370-2693(00)00359-2

[12] Huang, Q.-G. Weak Gravity Conjecture with Large Extra Dimensions. http://arxiv.org/pdf/hep-th/0610106.pdf

[13] Ng, Y.J. (2008) Space-Time Foam: From Entropy and Holography to Infinite Statistics and Nonlocality. Entropy, 10, 441-461.

[14] Abbott, B.P., et al., LIGO Scientific Collaboration and Virgo Collaboration (2016) Observation of Gravitational Waves from a Binary Black Hole Merger. Physical Review Letters, 116, Article ID: 061102. https://physics.aps.org/featured-article-pdf/10.1103/PhysRevLett.116.061102

[15] Valev, D. Neutrino and Graviton Rest Mass Estimations by a Phenomenological Approach. https://arxiv.org/ftp/hep-ph/papers/0507/0507255.pdf

\section{Submit or recommend next manuscript to SCIRP and we will provide best service} for you:

Accepting pre-submission inquiries through Email, Facebook, LinkedIn, Twitter, etc. A wide selection of journals (inclusive of 9 subjects, more than 200 journals)

Providing 24-hour high-quality service

User-friendly online submission system

Fair and swift peer-review system

Efficient typesetting and proofreading procedure

Display of the result of downloads and visits, as well as the number of cited articles

Maximum dissemination of your research work

Submit your manuscript at: http://papersubmission.scirp.org/

Or contact jhepgc@scirp.org 